\title{
Comparison with simulations to experimental data for photo- neutron reactions using SPring-8 Injector
}

\author{
Yoshihiro Asano ${ }^{1, *}$ \\ ${ }^{1}$ XFEL/SPring-8 Center, RIKEN 1-1 Koto Sayo Hyogo 679-5148, Japan
}

\begin{abstract}
Simulations of photo-nuclear reactions by using Monte Carlo codes PHITS and FLUKA have been performed to compare to the measured data at the SPring- 8 injector with $250 \mathrm{MeV}$ and $961 \mathrm{MeV}$ electrons. Measurement data of Bismuth-206 productions due to photo-nuclear reactions of ${ }^{209} \operatorname{Bi}(\gamma, 3 \mathrm{n})$ ${ }^{206} \mathrm{Bi}$ and high energy neutron reactions of ${ }^{209} \mathrm{Bi}(\mathrm{n}, 4 \mathrm{n}){ }^{206} \mathrm{Bi}$ at the beam dumps have been compared with the simulations. Neutron leakage spectra outside the shield wall are also compared between experiments and simulations.
\end{abstract}

\section{Introduction}

Since 1997 , the $8 \mathrm{GeV}$ class synchrotron radiation facility, SPring-8, has been operated successfully for the users. The shielding designs of the SPring- 8 accelerators were performed by using Jenkins' formula [1] for the bulk shielding in the lateral direction and modified Swanson's formula [2] in the forward direction. To verify the verification of the shielding design, benchmark experiments were performed by using Helium-3 high sensitive neutron counter with polyethylene moderators, TLD dose meters and Polymeric track detectors. The dose distributions within the shield tunnel were also measured by using $\mathrm{LiF}$ Thermo-Luminesence detectors and Polymetric neutron track detectors ( CR-39) and the data were presented at ICRS9 [3]. The high energy neutron distributions around the beam dump of the accelerated electron are also measured by using bismuth detectors. Those experimental data were presented and compared with the empirical formula and EGS4 [4] with photo-neutron production cross section data [5].

In addition to FLUKA [6,7], the Monte Carlo code PHITS2.82 [8,9] have been improved recently to simulate the photo-nuclear reaction calculation. These simulation codes are much useful for radiation shielding design of electron accelerator machines as well as proton accelators with good usability. The neutrons and gamma dose distributions around the beam dumps of the SPring8 injector have been simulated by using both Monte Carlo codes. However experimental data, especially photonuclear reaction data are quite insufficient to compare to the simulations.

Bismus is a useful metal to measure high energy neutrons and gamma-rays because of the neutron and gamma energy dependence of the nuclear production of the bithmus radioisotopes. At ICRS9, the data of bismus-
206 due to $250 \mathrm{MeV}$ electrons were presented. In addition to the data produced by $250 \mathrm{MeV}$ electrons, the experimental data produced by $961 \mathrm{MeV}$ electrons are presented and both data have been compared to simulations. The neutron spectra outside the shield tunnel are compared between the PHITS simulations and experimental data.

\section{Experiments}

The measurements were performed in 1998. At that time, the injector of SPring-8 had two dumps, one was $250 \mathrm{MeV}$ (L1) dump and the other was $1.15 \mathrm{GeV}$ (L2) dump. The geometrical configuration is shown in Fig.1. At the experiments, the $250 \mathrm{MeV}$ and $961 \mathrm{MeV}$ electron beam that lead to L1 dump and L2 dump were employed and the data are indicated in Table 1, respectively. The beam dumps have double cylindrical structures, made of graphite for the inner layer and iron for the outer layer to reduce photo-neutron production and shielding.

The neutron leakage spectra outside the shield tunnel were measured by using extremely high sensitive helium-3 counter with polyethylene moderators, and the data were unfolded with SAND-II code [10]. Two types (small and large) of bismuth ingot were set around the beam dumps to measure the high energy neutrons and gamma-rays.

Table 1. Electron beam conditions of the linac injector during

\begin{tabular}{|c|c|c|}
\multicolumn{4}{|c}{ the experiments. } \\
\hline Dump & $\begin{array}{c}\mathbf{2 5 0 M e V} \\
\text { (L1 dump) }\end{array}$ & $\begin{array}{c}\mathbf{1 . 1 5 G e V} \\
\text { (L2dump) }\end{array}$ \\
\hline Electron energy & $250 \mathrm{MeV}$ & $961 \mathrm{MeV}$ \\
\hline Frequency & $60 \mathrm{pps}$ & $60 \mathrm{pps}$ \\
\hline Peak current (40ns) & $143.0 \mathrm{~mA}$ & $134.3 \mathrm{~mA}$ \\
\hline Total electrons & $3.48 \times 10^{6}$ & $7.75 \times 10^{15}$ \\
\hline
\end{tabular}




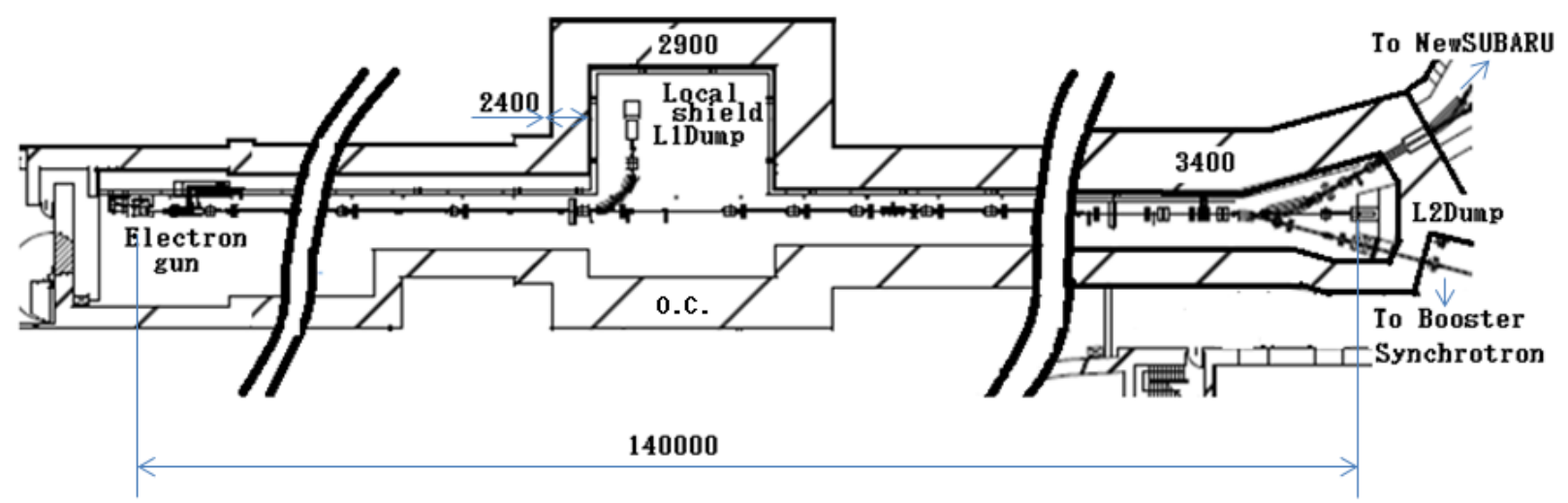

Fig. 1. Conceptual plane view of SPring-8 injector. (L1Dump and L2Dump are 250MeV dump and 1.15GeV dump, respectively. Unit is $\mathrm{mm}$ ) At the end station of the linac, accelerated electrons can be extracted to 3 ways. One is to L2 dump, one is to booster synchrotron, and the other is to small SR ring, NewSUBARU.

\section{Simulations}

Neutron and gamma effective dose (Worst geometry) simulations around the L1 dump by using FLUKA are shown in Figs.2 and 3, respectively. PHITS simulations for effective dose distribution (AP geometry) due to 961 $\mathrm{MeV}$ are shown in Figs. 4 and 5 for neutron and gamma doses, respectively.

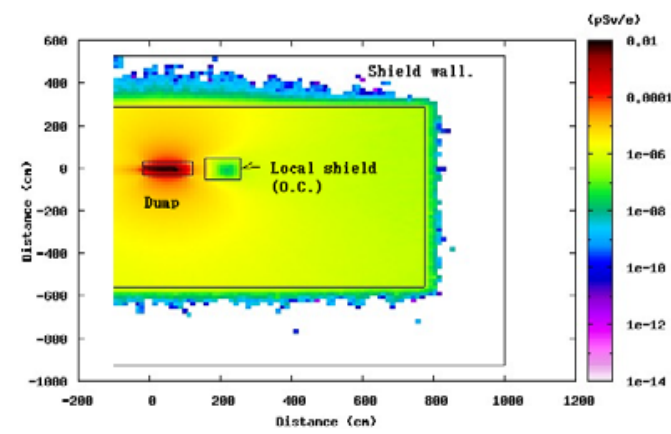

Fig.2. Neutron dose distribution at L1 dump (Horizontal plan at electron beam level, unit:pSv/e)

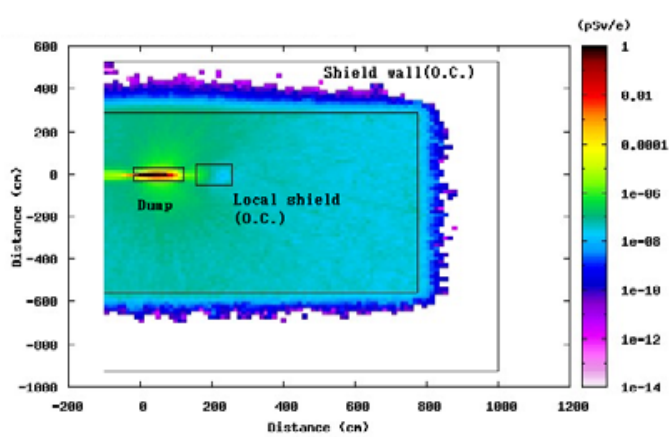

Fig.3. Gamma dose distribution at L1 dump. (Horizontal plan at electron beam level, unt;pSv/e)

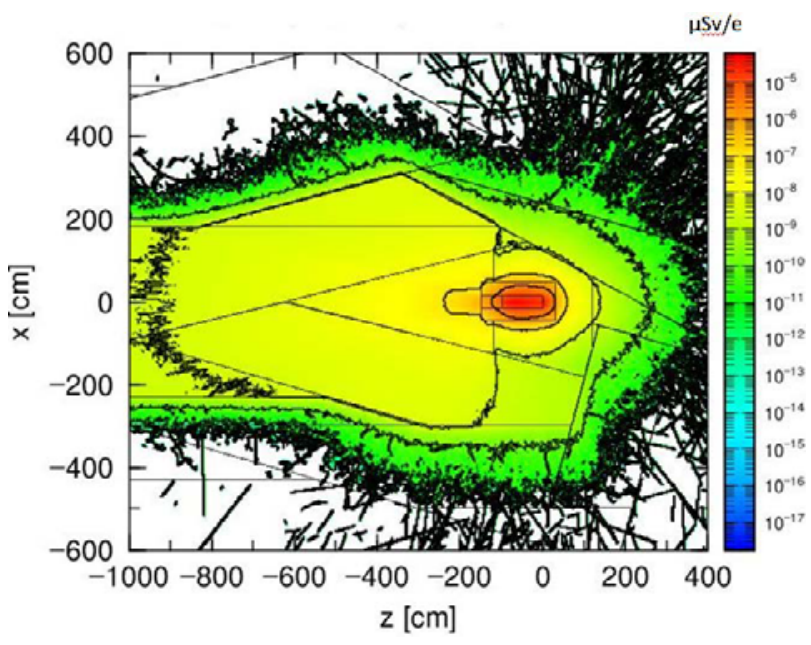

Fig.4. Neutron dose distribution due to $961 \mathrm{MeV}$ electrons. (Horizontal plane at electron beam level, unit: $\mu \mathrm{Sv} / \mathrm{e}$ )

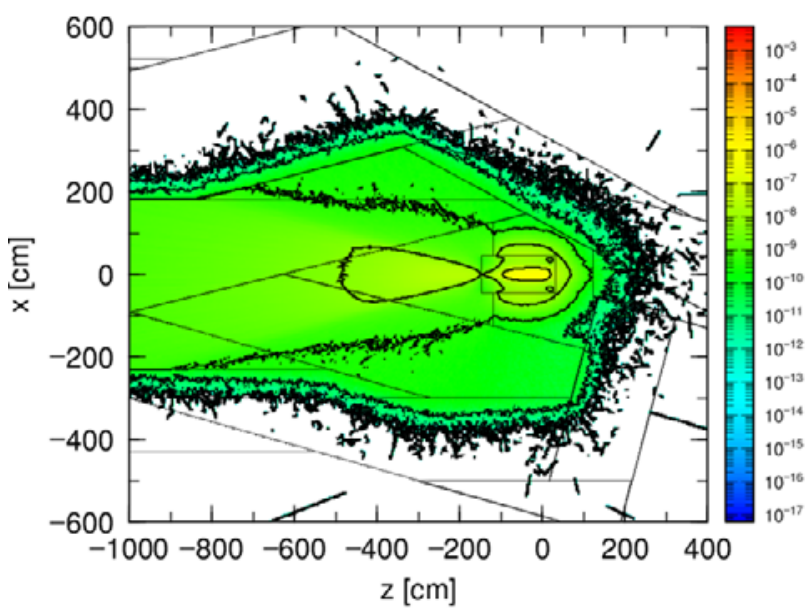

Fig.5. Gamma dose distribution due to $961 \mathrm{MeV}$ electrons. (Horizontal plane at electron beam level, unit: $\mu \mathrm{Sv} / \mathrm{e}$ ) 


\section{Comparison between simulations and experiments}

\subsection{Bismuth isotopes production around the beam dumps}

Small and large size bismuth ingots were employed for activation detectors and gamma-rays from bismuth detectors were measured after irradiation. The size of small detectors is $1.12 \mathrm{~cm}$ and $2.9 \mathrm{~cm}$ for the thickness and the diameter, respectively. The large detectors are $2.3 \mathrm{~cm}$ thick and $8 \mathrm{~cm}$ in diameter. In those cases, the self-shielding effect of detectors cannot be ignored to measure the gamma-rays. Figure 6 shows the selfshielding factors depending on gamma-ray energy and the size of bismuth detectors. These are PHITS simulations on the assumption of the isotropic distribution of the radioisotopes within the detectors. The assumption of the isotropic distribution is reasonable because of not so much thick detectors for high energy photons and neutrons and it's confirmed by using PHITS simulations. The previous data [3] were without

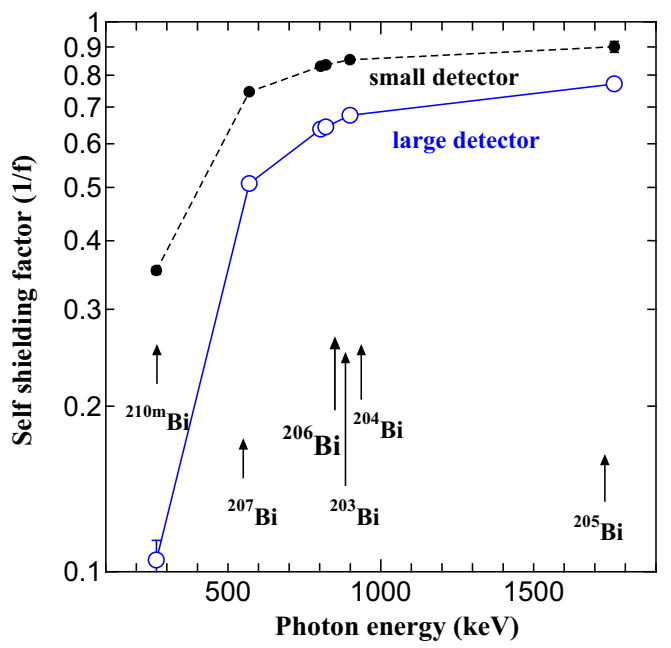

Fig.6. Self-shielding effect for the gamma-ray measurements of the bismuth radioisotopes depending on the photon energy and size of the detectors. The arrows of bismuth radioisotopes indicate the photon energy of main emissions. Full and open circles are for small and large detectors, respectively.

considering these self-shielding effects so that these data must be corrected, especially for large detectors. The PHITS simulations of bismuth isotopes productions have been performed using [T-Dchain] command and DCHAIN-SP [11] with weighting factor pnimul $=100$ of photo-nuclear reaction.

\subsubsection{MeV electrons (L1 dump)}

The bismuth-206 production distribution around the L1 dump was measured and compared to the Monte Carlo simulations. The bismuth detectors were set up as shown in Fig.7. In the experimental paper [3], the bismuth-206 production calculations were performed by using EGS4 with the library for ${ }^{209} \mathrm{Bi}(\gamma, 3 \mathrm{n}){ }^{206} \mathrm{Bi}$ reaction created by themselves using MCPHOTO [12] + PICA [13]. The reference paper also considered for ${ }^{209} \mathrm{Bi}(\mathrm{n}, 4 \mathrm{n}){ }^{206} \mathrm{Bi}$ reaction by using ENDF-B/VI library.

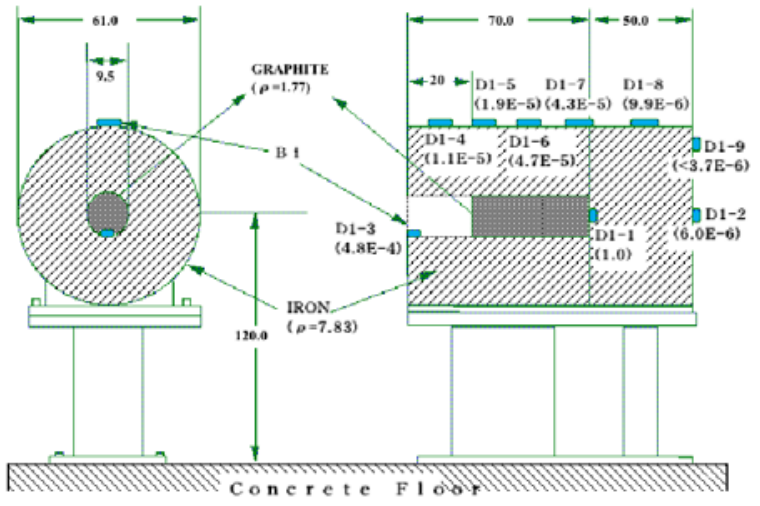

Fig.7. Illustration of the $250 \mathrm{MeV}$ electron beam dump (L1) and the location of the bismuth detectors. D1-1 to D1-9 are bismuth detectors and the figures in parenthesis under the detector number are the relative values of produced bismuth206, which are normalized to the D1-1 detector values [3].

Table 2. Bismuth-206 production distribution due to $250 \mathrm{MeV}$ electron injection into dump. The errors are considered only for statistical errors. The figures in the parentheses are pro forma amounts because of large statistical errors. (*; without considering self-shielding, *2; with considering self-shielding, *3; unit is converted from $\left(\mathrm{g}^{-1}\right.$ electron $\left.{ }^{-1}\right)[3]$ to $(\mathrm{Bq} / \mathrm{g}) /(\mathrm{e} / \mathrm{s})$

\begin{tabular}{|c|c|c|c|c|c|}
\hline Bi detector & $\begin{array}{c}\text { Measured data* } \\
(\mathrm{Bq} / \mathrm{g}) /(\mathrm{e} / \mathrm{s})\end{array}$ & $\begin{array}{c}\text { Measured data } \\
(\mathrm{Bq} / \mathrm{g}) /(\mathrm{e} / \mathrm{s})\end{array}$ & $\begin{array}{c}\text { EGS4 cal. } \\
(\mathrm{Bq} / \mathrm{g}) /(\mathrm{e} / \mathrm{s})\end{array}$ & $\begin{array}{c}\text { FLUKA cal. } \\
(\mathrm{Bq} / \mathrm{g}) /(\mathrm{e} / \mathrm{s})\end{array}$ & $\begin{array}{c}\text { PHITS cal. } \\
(\mathrm{Bq} / \mathrm{g}) /(\mathrm{e} / \mathrm{s})\end{array}$ \\
\hline D1-1(Small) & $1.45 \times 10^{-7} \mp 0.36 \%$ & $1.74 \times 10^{-7} \mp 0.36 \%$ & $3.7 \times 10^{-7}$ & $2.27 \times 10^{-7} \mp 0.50 \%$ & $2.21 \times 10^{-7} \mp 1.2 \%$ \\
\hline D1-2(Small) & $8.47 \times 10^{-13} \mp 25 \%$ & $1.02 \times 10^{-12} \mp 25 \%$ & $6.0 \times 10^{-12}$ & $\left(6.65 \times 10^{-12} \mp 14 \%\right)$ & - \\
\hline D1-3(Small) & $6.92 \times 10^{-11} \mp 2.3 \%$ & $8.34 \times 10^{-11} \mp 2.3 \%$ & $2.8 \times 10^{10}$ & $\left(1.40 \times 10^{-11} \mp 9.6 \%\right)$ & $\left(1.70 \times 10^{-10} \mp 40 \%\right)$ \\
\hline D1-4(Large) & $1.61 \times 10^{-12} \mp 2.9 \%$ & $2.52 \times 10^{-12} \mp 2.9 \%$ & $1.2 \times 10^{-12}$ & $\left(2.60 \times 10^{-12} \mp 14 \%\right)$ & - \\
\hline D1-5(Large) & $2.75 \times 10^{-12} \mp 2.2 \%$ & $4.31 \times 10^{-12} \mp 2.2 \%$ & $6.2 \times 10^{-12}$ & $5.03 \times 10^{-12} \mp 3.7 \%$ & $\left(2.38 \times 10^{-10} \mp 23 \%\right)$ \\
\hline D1-6(Large) & $6.79 \times 10^{-12} \mp 1.4 \%$ & $1.06 \times 10^{-11} \mp 1.4 \%$ & $1.4 \times 10^{-12}$ & $1.30 \times 10^{-11} \mp 2.7 \%$ & - \\
\hline D1-7(Large) & $6.21 \times 10^{-12} \mp 1.5 \%$ & $9.74 \times 10^{-12} \mp 1.5 \%$ & $7.5 \times 10^{-12}$ & $1.30 \times 10^{-11} \mp 4.5 \%$ & $\left(1.36 \times 10^{-10} \mp 41 \%\right)$ \\
\hline D1-8(Large) & $1.40 \times 10^{-12} \mp 3.1 \%$ & $2.19 \times 10^{-12} \mp 3.1 \%$ & $2.9 \times 10^{-12}$ & $\left(1.78 \times 10^{-12} \mp 14 \%\right)$ & - \\
\hline D1-9(Small) & $<5.35 \times 10^{-13}$ & $<6.45 \times 10^{-13}$ & $5.2 \times 10^{-14}$ & - & - \\
\hline
\end{tabular}


Table 2 indicates the results of the FLUKA and PHITS simulations together with the measurement data and the EGS4 calculations. In the simulations, FLUKA indicates about 1.30 times higher than that of the measurement data of D1-1 detector. The FLUKA simulation shows close agreement with the PHITS results for the bismuth206 production within the D1-1 detector. For another detecors except D1-2 and D1-3, the FLUKA simulations show good agreement within about 30\% discrepancies with the measured data. Large stastistical errors are indicated for the D1-2, D1-3 detectors, and the PHITS simulations have also large stastical errors for another detectors. Experimental data of another bismuth radioisotopes with the self shielding corrections are shown in Table 3 including PHITS simulations for D1-1 detector. In Table 3, «unknown» means that a long decay time was needed to measure the gamma-rays because of very high activities. Therefore, the information of bismuth-204 and 203 was cleared.

Table 3. Bismuth radioisotopes production within D1-1 and D1-6 detecors. (* ; measurement data)

\begin{tabular}{|c|c|c|c|}
\hline $\begin{array}{c}\text { Bi-det. } \\
\mathrm{No}\end{array}$ & $\begin{array}{c}\text { Exp.(D1-1)* } \\
(\mathrm{Bq} / \mathrm{g}) /(\mathrm{e} / \mathrm{s})\end{array}$ & $\begin{array}{c}\text { PHITS Cal } \\
(\mathrm{D} 1-1) \\
(\mathrm{Bq} / \mathrm{g}) /(\mathrm{e} / \mathrm{s})\end{array}$ & $\begin{array}{c}\text { EXP.(D1-6)* } \\
(\mathrm{Bq} / \mathrm{g}) /(\mathrm{e} / \mathrm{s})\end{array}$ \\
\hline${ }^{207} \mathrm{Bi}$ & $\begin{array}{c}2.68 \times 10^{-6} \\
\mp 6.4 \%\end{array}$ & $\begin{array}{c}3.66 \times 10^{-6} \\
\mp 0.22 \%\end{array}$ & $<2.37 \times 10^{-10}$ \\
\hline${ }^{205} \mathrm{Bi}$ & $\begin{array}{c}1.01 \times 10^{-7} \\
\mp 1.0 \%\end{array}$ & $\begin{array}{c}2.52 \times 10^{-8} \\
\mp 1.4 \%\end{array}$ & $\begin{array}{c}9.95 \times 10^{-12} \\
4.8 \%\end{array}$ \\
\hline${ }^{204} \mathrm{Bi}$ & unknown & - & $\begin{array}{c}8.94 \times 10^{-13} \\
16 \%\end{array}$ \\
\hline${ }^{203} \mathrm{Bi}$ & unknown & - & $\begin{array}{c}1.24 \times 10^{-12} \\
31 \%\end{array}$ \\
\hline
\end{tabular}

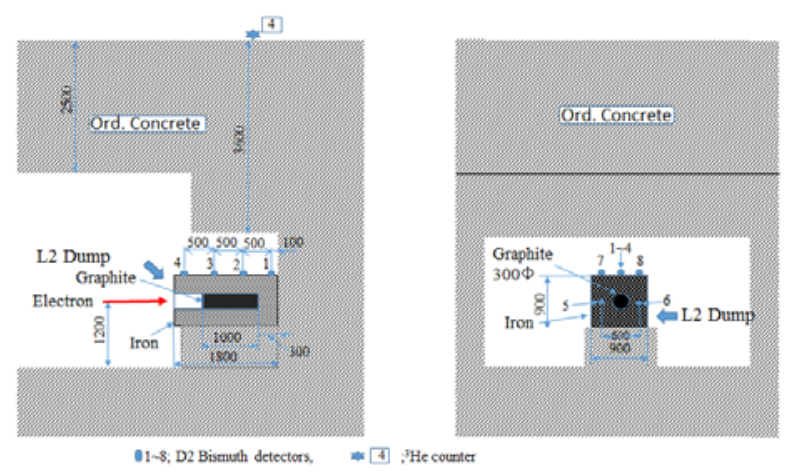

Fig.8. Configuration of bismuth detectors around the L2 beam dump (Vertical plane, left figure is parallel and right is perpendicular against the electron beam axis )

\subsubsection{MeV electrons (L2 dump)}

The bismuth-206 production distribution around the L2 dump using $961 \mathrm{MeV}$ electrons was measured as well as $250 \mathrm{MeV}$ electrons, and the configuration of bismuth detectors are as shown in Fig.8. The measurement data and simulation results are listed in Table 4. Both simulations show agreement within about $\mp 50 \%$ as shown in Fig.9 even though simulations have large stastical errors. Another bismuth radioisotopes which were produced in the detectors of D2-2, D2-3, and D2-4 are listed in Table 5 including the PHITS simulations for D2-3 detector. The measurement data of Tables 2 to 5 are useflu to estimate the photon and neutron energy distributions because of the threshold energy dependence on the photon and neutron energy for each reactions such as $22.44 \mathrm{MeV}, 29.48 \mathrm{MeV}, 37.97 \mathrm{MeV}$, and $45.16 \mathrm{MeV}$ for ${ }^{209} \mathrm{Bi}(\gamma, 3 \mathrm{n}){ }^{206} \mathrm{Bi},{ }^{209} \mathrm{Bi}(\gamma, 4 \mathrm{n}){ }^{205} \mathrm{Bi},{ }^{209} \mathrm{Bi}(\gamma, 5 \mathrm{n}){ }^{204} \mathrm{Bi}$, and ${ }^{209} \mathrm{Bi}(\gamma, 6 \mathrm{n}){ }^{203} \mathrm{Bi}$, respectively. It is difficult to estimate the amount of ${ }^{210} \mathrm{Bi}$ experimentally and directly, however it is easy to compare between the simulations. In comparison with the simulations, ${ }^{210} \mathrm{Bi}$ productions are listed as indicated in Table 6 , and shown in good agreements.

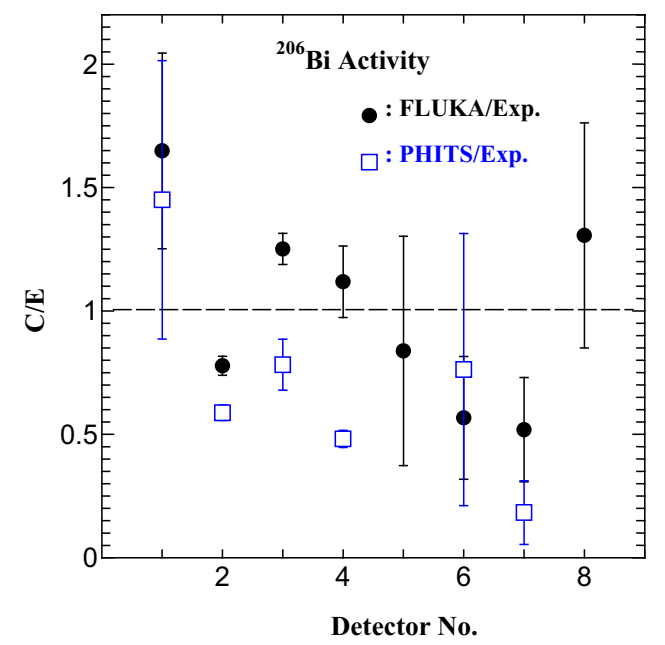

Fig.9. Ratios of simulations to experimental data (Full circles and open squres are the data of FLUKA and PHITS, respectively. The errors are only considering stastical errors.)

\subsection{Leakage neutron spectrum outside the roof of shield tunnel}

Leakage neutron spectra have been simulated by using PHITS with the [T-Point] tally of which algorism is same as the Point Detector of MCNP5 [14] because of strong attenuation through thick shield walls. In these simulations, two points have been performed to compare the experimental data [3]. One is located directly above the L1 dump outside the roof of the tunnel with the shield thickness of $190 \mathrm{~cm}$ ordinary concrete, and the other is located directly above the L2 dump outside the roof of the tunnel with the shield thickness of $360 \mathrm{~cm}$ ordinary concrete as indicated in Figs. 1 and 8 . These points are under air conditioning so that it can be avoided the change of the environmental conditions, relatively. In the simulations, the density of $2.26 \mathrm{~g} / \mathrm{cm}^{3}$ has been employed for ordinary concrete. Figs. 10 and 11 show the simulation results in comparison with the experimental data that were measured by using extremely high sensitive helium-3 counter with multipolyethylene moderators and unfolded the measurement data. These simulations show reasonably agree with the experimental data. However, the results of the simulation for the $250 \mathrm{MeV}$ electron case indicate slightly high in the region of high energy in comparison with the 
experimental data as shown in Fig.10. On the other hand, Fig. 11 shows that the simulation results for the $961 \mathrm{MeV}$ electron case indicate the slightly lower spectrum than that of experimental data in the region of intermediate

Table 4 Bismuth-206 production distribution due to $961 \mathrm{MeV}$ electron injection into L2 dump. The errors are considered only for statistical errors. The figures in the parentheses are pro forma amounts because of large statistical errors. (*; without considering self-shielding, $* 2$; with considering self-shielding, *3; unit is converted from $\left(\mathrm{g}^{-1}\right.$ electron $\left.{ }^{-1}\right)[3]$ to $(\mathrm{Bq} / \mathrm{g}) /(\mathrm{e} / \mathrm{s})$

\begin{tabular}{|c|c|c|c|c|c|c|}
\hline $\begin{array}{c}\text { Bi detector } \\
\text { No. }\end{array}$ & $\begin{array}{c}\text { Measured data* } \\
(\mathrm{Bq} / \mathrm{g}) /(\mathrm{e} / \mathrm{s})\end{array}$ & $\begin{array}{c}\text { Measured data } \\
(\mathrm{Bq} / \mathrm{g}) /(\mathrm{e} / \mathrm{s})\end{array}$ & $\begin{array}{c}\text { FLUKA cal. } \\
(\mathrm{Bq} / \mathrm{g}) /(\mathrm{e} / \mathrm{s})\end{array}$ & $\begin{array}{c}\text { PHITS cal. } \\
(\mathrm{Bq} / \mathrm{g}) /(\mathrm{e} / \mathrm{s})\end{array}$ & $\begin{array}{c}\text { C/E } \\
(\mathrm{FLUKA})\end{array}$ & $\begin{array}{c}\text { C/E } \\
(\mathrm{PHITS})\end{array}$ \\
\hline $\mathrm{D} 2-1($ Small) & $1.08 \times 10^{-11} \mp 17 \%$ & $1.31 \times 10^{-11} \mp 17 \%$ & $\left(2.16 \times 10^{-11} \mp 17 \%\right)$ & $\left(1.90 \times 10^{-11} \mp 35 \%\right)$ & $(1.7 \mp 24 \%)$ & $(1.5 \mp 39 \%)$ \\
\hline $\mathrm{D} 2-2($ Large $)$ & $2.01 \times 10^{-11} \mp 1.8 \%$ & $3.15 \times 10^{-11} \mp 1.8 \%$ & $2.45 \times 10^{-11} \mp 4.6 \%$ & $1.85 \times 10^{-11} \mp 5.0 \%$ & $0.78 \mp 4.9 \%$ & $0.59 \mp 5.3 \%$ \\
\hline $\mathrm{D} 2-3($ Large $)$ & $1.14 \times 10^{-11} \mp 2.3 \%$ & $1.79 \times 10^{-11} \mp 2.3 \%$ & $2.24 \times 10^{-11} \mp 4.5 \%$ & $\left(1.40 \times 10^{-11} \mp 13 \%\right)$ & $1.3 \mp 5.1 \%$ & $(0.78 \mp 13 \%)$ \\
\hline $\mathrm{D} 2-4($ Large $)$ & $1.56 \times 10^{-12} \mp 6.8 \%$ & $2.45 \times 10^{-12} \mp 6.8 \%$ & $2.74 \times 10^{-12} \mp 11 \%$ & $1.18 \times 10^{-12} \mp 2.2 \%$ & $1.1 \mp 13 \%$ & $0.48 \mp 7.1 \%$ \\
\hline $\mathrm{D} 2-5($ Small) & $3.50 \times 10^{-12} \mp 24 \%$ & $4.26 \times 10^{-12} \mp 24 \%$ & $\left(3.57 \times 10^{-12} \mp 50 \%\right)$ & - & $(0.84 \mp 55 \%)$ & - \\
\hline $\mathrm{D} 2-6($ Small) & $5.22 \times 10^{-12} \mp 18 \%$ & $6.28 \times 10^{-12} \mp 18 \%$ & $\left(3.56 \times 10^{-12} \mp 40 \%\right)$ & $\left(4.79 \times 10^{-12} \mp 70 \%\right)$ & $(0.57 \mp 44 \%)$ & $(0.76 \mp 72 \%)$ \\
\hline $\mathrm{D} 2-7($ Large $)$ & $1.09 \times 10^{-12} \mp 7.8 \%$ & $1.71 \times 10^{-12} \mp 7.8 \%$ & $\left(8.87 \times 10^{-13} \mp 40 \%\right)$ & $\left(3.13 \times 10^{-13} \mp 13 \%\right)$ & $(0.52 \mp 41 \%$ & $(0.18 \mp 70 \%)$ \\
\hline D2-8(Large) & $1.02 \times 10^{-12} \mp 8.0 \%$ & $1.60 \times 10^{-21} \mp 8.0 \%$ & $2.09 \times 10^{-12} \mp 34 \%$ & $\left(1.40 \times 10^{-11} \mp 13 \%\right)$ & $(1.31 \mp 35 \%)$ & - \\
\hline
\end{tabular}

Table 5 Bismuth radioisotopes production within D2-2, 3, and 4 detecors. (uni ;(Bq/g)/(e/s), *; measurement data)

\begin{tabular}{|c|c|c|c|c|}
\hline $\mathrm{Bi}$ & $(\mathrm{D} 2-2)^{*}$ & $(\mathrm{D} 2-3)^{*}$ & $\begin{array}{c}\text { PHITS Cal. } \\
(\mathrm{D} 2-3)\end{array}$ & $(\mathrm{D} 2-4)^{*}$ \\
\hline${ }^{205} \mathrm{Bi}$ & $\begin{array}{c}3.22 \times 10^{-11} \\
\mp 6.6 \%\end{array}$ & $\begin{array}{c}1.86 \times 10^{-11} \\
\mp 8.2 \%\end{array}$ & $\begin{array}{c}1.52 \times 10^{-11} \\
\mp 36 \%\end{array}$ & $\begin{array}{c}3.44 \times 10^{-12} \\
\mp 18 \%\end{array}$ \\
\hline \multirow{2}{*}{${ }^{204} \mathrm{Bi}$} & $\begin{array}{c}1.89 \times 10^{-11} \\
\mp 3.1 \%\end{array}$ & $\begin{array}{c}9.13 \times 10^{-12} \\
\mp 4.0 \%\end{array}$ & $\begin{array}{c}1.16 \times 10^{-11} \\
\mp 46 \%\end{array}$ & $\begin{array}{c}1.52 \times 10^{-12} \\
\mp 13 \%\end{array}$ \\
\hline${ }^{203} \mathrm{Bi}$ & $\begin{array}{c}1.65 \times 10^{-11} \\
\mp 6.1 \%\end{array}$ & $\begin{array}{c}9.51 \times 10^{-12} \\
\mp 8.4 \%\end{array}$ & $\begin{array}{c}3.48 \times 10^{-12} \\
\mp 17 \%\end{array}$ & $\begin{array}{c}6.42 \times 10^{-13} \\
\mp 55 \%\end{array}$ \\
\hline
\end{tabular}

Table 6 Simulation of bismuth-210 production

\begin{tabular}{|c|c|c|c|}
\hline $\begin{array}{c}\text { Det. } \\
\text { No }\end{array}$ & $\begin{array}{c}\text { FLUKA cal. } \\
(\mathrm{Bq} / \mathrm{g}) /(\mathrm{e} / \mathrm{s})\end{array}$ & $\begin{array}{c}\text { PHITS cal. } \\
(\mathrm{Bq} / \mathrm{g}) /(\mathrm{e} / \mathrm{s})\end{array}$ & $\begin{array}{c}\text { FLUKA cal } \\
\text { PHIT cal. }\end{array}$ \\
\hline No. 1 & $5.64 \times 10^{-11} \mp 1.7 \%$ & $4.22 \times 10^{-11} \mp 1.9 \%$ & $1.3_{4} \mp 2.6 \%$ \\
\hline No. 2 & $3.34 \times 10^{-11} \mp 1.6 \%$ & $4.34 \times 10^{-11} \mp 1.0 \%$ & $0.77_{0} \mp 1.9 \%$ \\
\hline No.3 & $\begin{array}{c}3.04 \times 10^{-11} \mp \\
0.88 \%\end{array}$ & $\begin{array}{c}2.97 \times 10^{-11} \mp \\
0.85 \%\end{array}$ & $1.0_{2} \mp 1.2 \%$ \\
\hline No.4 & $9.99 \times 10^{-12} \mp 1.9 \%$ & $1.05 \times 10^{-11} \mp 3.2 \%$ & $0.95_{1} \mp 3.7 \%$ \\
\hline No.5 & $2.64 \times 10^{-12} \mp 6.4 \%$ & $2.67 \times 10^{-12} \mp 1.7 \%$ & $0.98_{9} \mp 6.6 \%$ \\
\hline No.6 & $2.74 \times 10^{-12} \mp 9.6 \%$ & $3.24 \times 10^{-12} \mp 2.3 \%$ & $0.84_{6} \mp 9.9 \%$ \\
\hline No.7 & $8.60 \times 10^{-12} \mp 2.5 \%$ & $8.11 \times 10^{-12} \mp 6.1 \%$ & $1.0_{6} \mp 6.6 \%$ \\
\hline No.8 8 & $8.24 \times 10^{-12} \mp 2.0 \%$ & $7.70 \times 10^{-12} \mp 4.1 \%$ & $1.0_{7} \mp 4.6 \%$ \\
\hline
\end{tabular}

energy region. These discrepancies need future investigations as well as the improvements of the statistical errors.

\section{Summary}

The experimental data of bismuth radioisotopes distribution at around the beam dumps produced by 961 $\mathrm{MeV}$ electrons are presented as well as that of due to 250
$\mathrm{MeV}$ electrons. These data are useful to compare to the simulations. FLUKA and PHITS Monte Carlo simulations have been performed to compare to these data and the both simulations show reasonably agreements in a range of within about $\mp 50 \%$. The PHITS simulations are performed for the leakage neutron energy spectra to compare to the experimental data, and these show slightly different shapes. These discrepancies need future investigations.

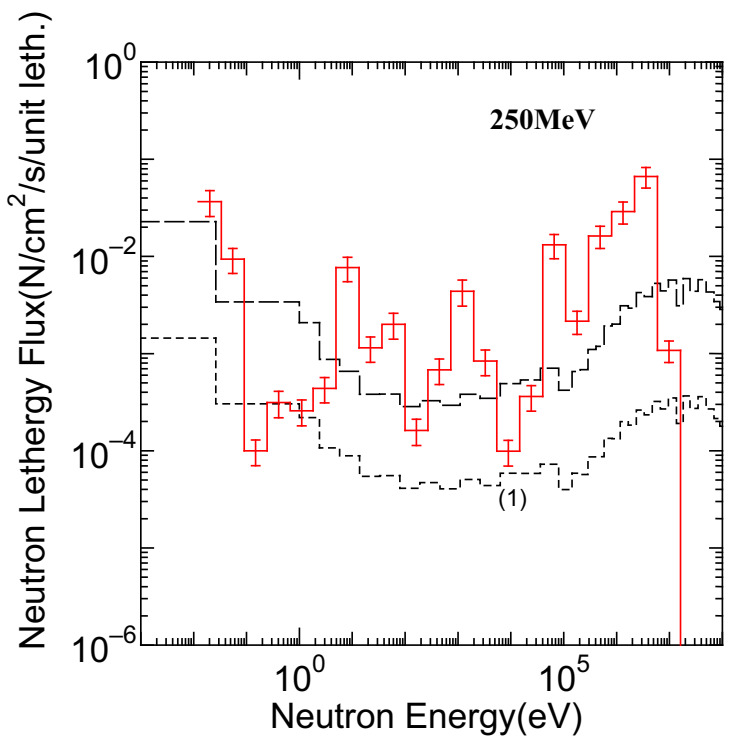

Fig.10 Comparison of the leakage neutron energy spectrum at the roof outside the shield tunnel and directly above of the L1 dump. (Solid red line indicates the simulation results, and dashed line is the experimental data. Dotted line indicates the neutron back ground spectrum) 


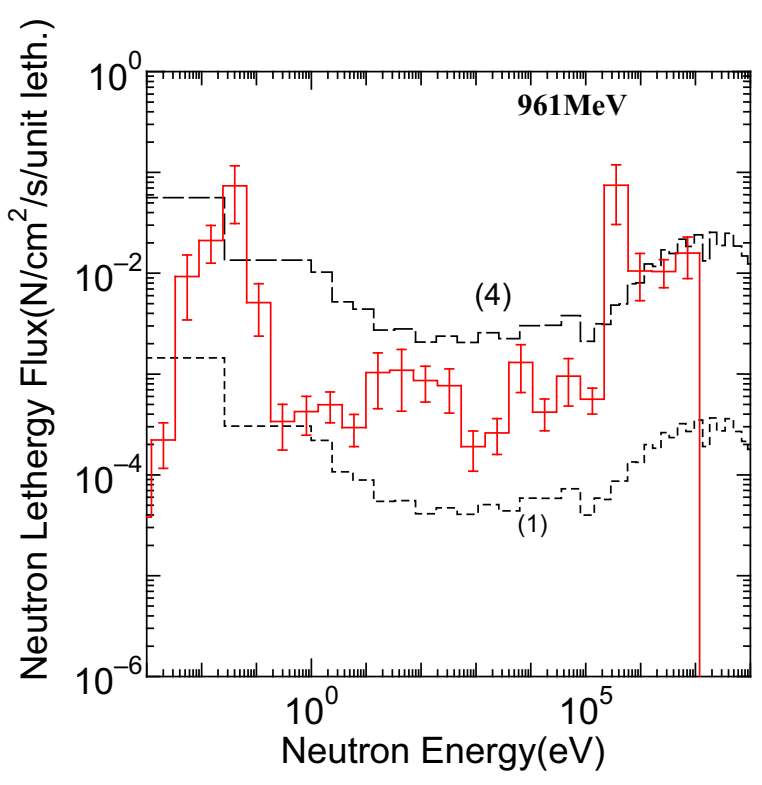

Fig.11 Comparison of the leakage neutron energy spectrum at the roof outside the shield tunnel and directly above of the L2 dump. Lines indicate the same as Fig.10.

The author wishes to thank Dr. Tatsuhiko.Sato of JAEA and the developer group members for thier help and valuable discussions to use PHITS.

\section{References}

1. T.M. Jenkins, Neutron and photon measurements through concrete from a $15 \mathrm{GeV}$ electron beam on a target-Comparison with models and calculations, Nuclear Instruments and Methods 159265 (1979)

2. E.Braeuer, Radiation shielding for the $6 \mathrm{GeV}, \mathrm{ESRF}$, ESRF/SHILED/88-04 (1988).

3. Y.Asano \& N.Sasamoto, Benchmark Experiment for Radiation Shielding around the Beam Dump of the SPring-8 Injector, J.of Nuclear Science and Technology Supple. 1 535-539 (2000)

4. W.R.Nelson et al. The EGS4 code system, SLAC-265 UC-32 (1985)

5. S.S.Dietrich et al. Atlas of photoneutron cross section obtained with monoenergetic photons, Atomic Data and Nucl. Data tables 38 (1988)

6. G.Battistoni et al. The FLUKA code : Discription and benchmarking, Proc. Of the Hadronic Shower Simulation Workshop 2006, AIP conf. proceeding 896,31-49 (2007)

7. A.Fasso et al. FLUKA a multi-particle transport code, CERN-2005-10 INFN/TC/05/11 SLAC-R-773 (2005)

8. T.Sato et al. Particle and heavy ion transport code system PHITS, version 2.52, J. Nucle. Sci. Technol. 50:9 913-923 (2013)

9. S.Noda et al. Improvement of photonuclear reaction model below $140 \mathrm{MeV}$ in the PHITS code, J. Nucle. Sci. and Technol,00223131.2014.923349, (2014)
10. P.J.Griffin et al. User's Manual for SNL-SAND-II Code, AFWL-TR 67-41, CCC-112 SAND93-3957 UC-713 (1969)

11. T.Kai et al. DCHAIN-SP 2001 :High energy particle induced radioactivity calculation code, JAERI-DataCode-2001-016 (2001) in Japanese

12. N.Kishida, Methods used in photonuclear data evaluation at JNDC, Proc. Int. Synposium on Nuclear Data Eval BNL 598 (1992)

13. T.A.Gabriel et al., PICA Monte Carlo MediumEnergy photon-induced intranuclear Cascade Analysis Code System, ORNL CCC-160 (1972)

14. X-5 Monte Carlo Team. MCNP-A General Monte Carlo N-Particle Transport Code, Version 5, LAUR-03-1987 (1987) 